\title{
NUCHAL CORD;
}

NUCHAL CORD PREVALENCE AND PERINATAL OUTCOME IN CASES PRESENTED IN KHYBER TEACHING HOSPITAL, PESHAWAR: A TERTIARY CARE HOSPITAL.

1. MBBS, FCPS (O \& G)

Assistant Professor

Department of Gynae B Unit

Khyber Teaching Hospital, Peshawa

2. MBBS, Trainee

Registrar

Department of Gynae B Unit,

Khyber Teaching Hospital, Peshawar.

Correspondence Address:

Dr. Abida Nasreen

Department of Gynae B Unit

Khyber Teaching Hospital, Peshawar.

abidaibrar@yahoo.com

Article received on:

31/01/2018

Accepted for publication:

15/10/2018

Received after proof reading:

$04 / 01 / 2019$

\section{Abida Nasreen', Hina Niaz ${ }^{2}$}

ABSTRACT... Objectives: To report nuchal cord prevalence and perinatal outcome in cases presented in a tertiary care hospital at Peshawar. Study Design: It is a cross sectional prospective study. Duration: The period of study was 18 months from 01.01. 2013 to 30.06 2014. Setting: Department of Obstetrics and Gynaecology, "C" unit of Khyber Teaching Hospital Peshawar. Methodology: In this study, 705 cases with nuchal cord were included out of 4746 cases. After admission detailed obstetrics history, general physical, abdominal, pervaginal examination, and pelvic assessment was also done. All the patients were closely observed for the feto-maternal outcomes. Maternal age, parity, mode of delivery, fetal distress, meconium presence in liquor and APGAR score, abnormal fetal heart rate, admission to neonatology unit, presence of nuchal cord at delivery, and other were noted. Results: A total of 705 (14.85\%) cases had nuchal cord. The single loop of cord was recorded in $63 \%$ cases, $50.63 \%$ cases were loose and diagnosed during delivery in $61.5 \%$ cases. FHR irregularities were in $18.29 \%$ cases in $28.5 \%$ cases recorded the meconium staining of liquor. APGAR score $<7$ at 1 -minute was in $28.22 \%$ cases. The maternal-age ranged $15-40$ years and majority (37.02\%) was multigravida. $50.49 \%$ the maximum cases in which normal vaginal delivery was done. Conclusion: Nuchal cord frequency was observed not higher as compared to reported previously. At the time of delivery, maximum cases were detected and minimum fetal adverse outcomes. The main mode of delivery was normal vaginal delivery.

Key words: $\quad$ Nuchal Cord-Frequency-Fetal-Adverse-Outcomes.

Article Citation: Nasreen A, Niaz H. Nuchal cord; nuchal cord prevalence and perinatal outcome in cases presented in khyber teaching hospital, Peshawar: a Tertiary Care Hospital. Professional Med J 2019; 26(1):109-115. DOI: 10.29309/TPMJ/2019.26.01.2583

\section{INTRODUCTION}

A nuchal cord occurs while the umbilical wire turns into wrapped 360 levels across the fetus neck. ${ }^{1}$ This is commonly found by ultrasonography before delivery or at delivery. The prevalence of nuchal cord at delivery, reported as $28.2-33.7 \%, 2,3$ apparently increases in prolonged gestations. ${ }^{4} \mathrm{~A}$ study based on 166,318 deliveries demonstrated that nuchal cord is linked with nonreassuring fetal heart rate (FHR) during labor. ${ }^{5}$ However, a higher rate of cesarean delivery was not observed in nuchal cord groups. ${ }^{6-8}$ Several studies also claimed that routine antenatal ultrasonography is not essential because nuchal cord isn't always related to unfavourable neonatal outcomes. . $^{2,3,7,8}$ Conversely, studies have demonstrated that umbilical cord complications are found in most stillbirths. ${ }^{9,10}$
Nkwabong and Fomulu ${ }^{11}$ reported a perinatal mortality of $6.1 \%$ in 198 cases of nuchal cord in a hospital in Cameroon, where clinicians have been not always privy to the presence of nuchal cord because ultrasonography was sometimes unavailable. A study examining cerebral palsy in infants reported that a tight nuchal cord is a risk factor for spastic cerebral palsy and spastic quadriplegia. $^{12}$ Although many studies have reported that a higher risk of nonreassuring FHR is found in fetuses with a nuchal cord, there is no consensus regarding perinatal risk management owing to different neonatal outcomes reported in the literature. ${ }^{13}$ Including nuchal cord as a risk factor for adverse perinatal outcomes without careful consideration might increase cesarean rates on patient's request due to maternal anxiety. ${ }^{14}$ 
Umbilical cord can affect the infant's popularity before hard work, in the course of parturition and after delivery even. It occurs in $20-30 \%$ of births. ${ }^{15} \mathrm{It}$ is believed by obstetricians to be the underlying cause of unexplained fetal distress, neonatal depression and has even attributed to be a frequent cause of perinatal morbidity. Some obstetricians clamp the cord immediately after delivery of the head and before the shoulders, whereas other do not clamp at all. ${ }^{15}$ Even though most babies seem to tolerate this manner with minimum misery, there's evidence that reducing the wire earlier than shipping of the shoulders can result in neonatal morbidity or even mortality. ${ }^{16}$

Latest prenatal ultrasonographic evaluation famous nuchal chords are dynamic in nature, forming and resolving over the direction of being pregnant. ${ }^{17}$ Even in 10 to 14 weeks gestation $8 \%$ of the fetuses may have nuchal cord present. ${ }^{18}$ The occurrence of the nuchal cord increases with gestational age at 42 weeks. $29 \%$ of births have nuchal cord present. ${ }^{15}$

\section{METHODOLOGY}

This was a prospective cross sectional study, conducted in Obs \& Gyne Unit "C" of Medical Teaching Institute, Khyber Hospital, Peshawar for 18 months from $1^{\text {st }}$ January 2013 to $30^{\text {th }}$ June 2014. A total of 13057 admissions were recorded and 4746 delivered at term during the study period. Out of 4746 cases, 705 cases which had nuchal cord were included. Women who have singleton pregnancy of greater than 28 weeks and $37-41$ weeks +6 days (full term) with the cephalic presentations were included. Patients with congenital anomaly, preterm delivery, chorioammionitis, multifetal pregnancy, severe anemia, preeclampsia which can independently cause adverse prenatal outcome were excluded from the study. All cases as per inclusion criteria were admitted in our units through OPD or emergency department of the hospital. After getting permission from Hospital Ethical Committee, data was collected all those cases were given informed consents. After admission a detailed history regarding duration of amenorrhea, gravidity and parity, duration of pregnancy and history of labour pains was taken. General physical examination, abdominal examination for fundal height, lie, presentation engagement, amount of liquor, estimated fetal weight, palpable uterine contraction and fetal heart rate, pervaginal examination for assessing cervical dilatation and effacement, presentation and pelvic assessment was also done. Bishop scoring was calculated. History of toxemia, hypertension, antepartum hemorrhage was taken. All routine blood investigation along with ultrasonography was done. Duration of latent and active phase was plotted on Partogram and augmentation was done where required. All the patients were closely observed with reference to the course of labor, intervention required, mode of delivery and feto-maternal outcome. Colour Doppler study was required for confirmation of diagnosis.

Fetal heart rate monitory was done with intermittent auscultation with stethoscope every 30 minutes in $1^{\text {st }}$ stage of labour and every 10 minutes in $2^{\text {nd }}$ stage. FHR irregularities (less than 110 per minutes and greater than 180 per minutes) for 10 minutes were considered as sign of fetal distress. Variables like meconium staining of liquor, transient fetal heart rate irregularities, fetal distress;. $\mathrm{A} / \mathrm{S}<7$ at 1 minute and 5 minutes and admission to the antenatal unit were taken as outcome variables. Data was entered and analyzed by SPSS- 18 .

\section{RESULTS}

During this 18 months study period total no. of admissions in our units was 13057 and 4746 delivered at term. Of the total deliveries 705 cases had nuchal cord making its incidence $14.85 \%$ of all the deliveries. Among these 705 cases, the incident of single loop of cord was recorded in $447(63.4 \%)$, while double, triple and quadruple loops were 206 (29.2\%), 48 (6.8\%) and 4 (0.6\%) respectively. Of the total cases of nuchal cord 705, 357 (50.63\%) were loose and 349 (49.3\%) were tight. Nuchal cord was diagnosed before labour in 271 (38.4\%) and during delivery in 434 $(61.5 \%)$ cases (Table-I).

Transient FHR irregularities was common in 129 (18.29\%) cases, similarly presence of meconium 
staining of liquor was also recorded in 201 (28.5\%) cases. Apgar score $<7$ at 1 minute was present in 199 (28.22\%) cases (Table-II).

Among the maternal demographic characteristics, maternal age analysis showed that 30 (4.25\%) were in the age range of 15-20 years, 257 (36.45\%) were between 21-25 years, 211 (29.92\%) of the mothers were between 26-30 years, 108 (15.31\%) were aged $31-35$ years and 99 (19.04\%) were in the age range of $36-40$ years. The youngest maternal age in the study was 15 years and the oldest age was 40 years. Mean age was $27.88 \pm 5.80$ years. Out of 705 cases, primigravida were 241 (34.18\%), multigravida were 261 (37.02\%) and grandmultigravida were 203 (28.79\%). Mode of delivery showed that normal vaginal delivery was done in majority of cases i.e. 356 (50.49\%) cases, followed by cesarean section in 260 (36.87\%) cases, instrumental deliveries in 85 (12.05\%) cases and breech deliveries in 04 (0.56\%) cases (Table-III).

\begin{tabular}{|l|c|c|}
\hline \multicolumn{1}{|c|}{ Variable } & No. \\
\hline Loops of nuchal cords \\
\hline Single loop & 447 & 63.4 \\
\hline Double loops & 206 & 29.2 \\
\hline Triple loops & 48 & 6.8 \\
\hline Quadruple loops & 4 & 0.6 \\
\hline Types of nuchal cords & \\
\hline Loose & 357 & 50.6 \\
\hline Tight & 349 & 49.4 \\
\hline Nuchal cord diagnosed & \\
\hline Before labour & 271 & 38.4 \\
\hline During delivery & 434 & 61.6 \\
\hline \multicolumn{2}{|c|}{ Table-l. Various characteristics of cases $(\mathrm{n}=705)$} \\
\hline
\end{tabular}

\begin{tabular}{|l|c|c|}
\hline \multicolumn{1}{|c|}{ Outcome } & No. & $\%$ \\
\hline Fetal Heart Rate irregularities & 129 & 18.4 \\
\hline Meconium stained liquor & 201 & 28.5 \\
\hline Apgar score & & \\
\hline$<7$ at 1 minute & 199 & 28.2 \\
\hline$<7$ at 5 minute & 68 & 9.6 \\
\hline ,Admission to neonatal unit & 256 & 36.3 \\
\hline \multicolumn{2}{|c|}{ Table-Il. Various neonatal outcomes $(\mathrm{n}=705)$} \\
\hline
\end{tabular}

\begin{tabular}{|l|c|c|}
\hline \multicolumn{1}{|c|}{ Variable } & No. & $\%$ \\
\hline Maternal age (years) & \multicolumn{1}{|c|}{} \\
\hline $15-20$ & 30 & 4.25 \\
\hline $21-25$ & 257 & 36.45 \\
\hline $26-30$ & 211 & 29.92 \\
\hline $31-35$ & 108 & 15.31 \\
\hline $36-40$ & 99 & 14.04 \\
\hline Gravidity & & \\
\hline Primigravida & 241 & 34.18 \\
\hline Multigravida & 261 & 37.02 \\
\hline Grandmultigravida & 203 & 28.79 \\
\hline Mode of delivery & \multicolumn{2}{|c|}{} \\
\hline Normal vaginal delivery & 356 & 50.49 \\
\hline Cesarean section & 260 & 36.87 \\
\hline Instrumental delivery & 85 & 12.05 \\
\hline Breech delivery & 4 & 0.56 \\
\hline Table-III. Various maternal characteristics (n=705) \\
\hline
\end{tabular}

\section{DISCUSSION}

At the time of delivery, nuchal cord is a frequent finding and umbilical cord wrapped around babies (25\%) neck. ${ }^{19}$ In the present study prevalence of nuchal cord was found to be $14.85 \%$ and majority $61.50 \%$ cases were diagnosed at the time of delivery, which is not higher than reported in the national and international literature. These variations could be due to large or less number of sample size selected in various study. The presence of a nuchal cord at beginning occurs often sufficient to be taken into consideration a regular event. Studies document the prevalence of a nuchal cord as between $10 \%$ to $37 \%$. $^{2,4-7,20}$ But studies conducted in India observed fees as low as $2.1 \%,{ }^{21}$ and $5.74 \% .{ }^{22}$

A study of Greenwood and Impey ${ }^{23}$ compared outcomes associated with the presence of a good nuchal cord among one hospital that mechanically recorded the presence of a nuchal twine at each delivery and hospitals in which recording became at the discretion of the birth attendant. Whilst recording changed into discretionary the presence of a nuchal cord was related to lower apgar rankings and cerebral palsy. However, this affiliation turned into absent whilst nuchal cords were automatically documented. 


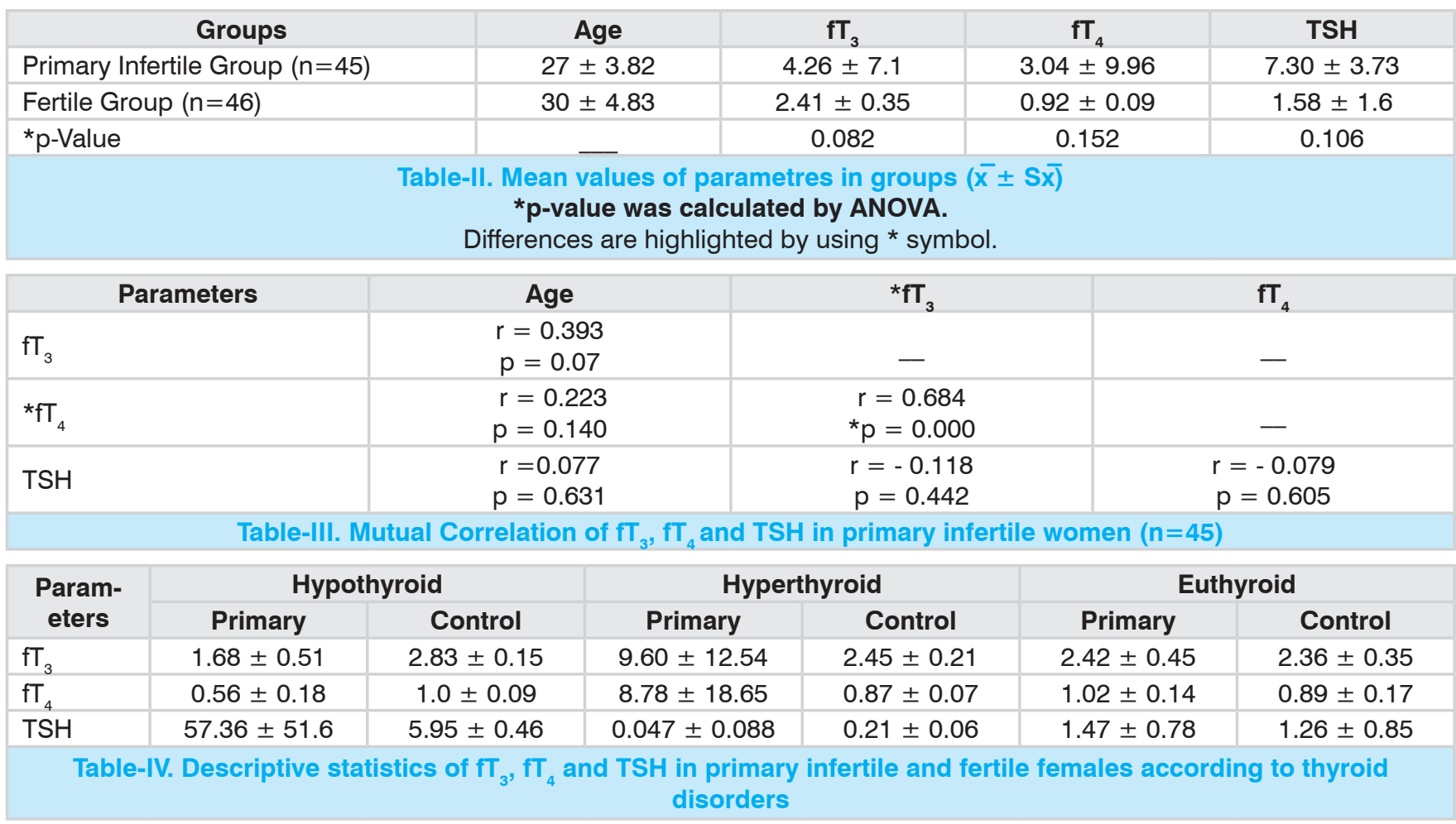

Those findings endorse that start attendants are more likely to record the presence of a nuchal cord while there's proof of neonatal compromise.

Different studies did no longer distinguish between a free nuchal cord and a good nuchal cord. 2,4,20 The studies that did differentiate observed the affiliation with complications changed into handiest related to tight nuchal cords. ${ }^{12,22}$ In our study loose nuchal cord was found in 50.63\% cases and tight nuchal cord was recorded in $49.30 \%$ cases.

In $20 \%$ to $33 \%$ term gestations there is one loop of cord around neck is present. The cord around neck risk increased from $5.8 \%$ (at 20 weeks) to $29 \%$ (at 42 weeks) as stated by a study. With the help of color Doppler ultrasound nuchal cord can be detected antenatal with $90 \%$ sensitivity after thirty six weeks of gestation. ${ }^{19}$ In our study one loop was found in maximum cases i.e. $63 \%$, which is higher than reported above and could be due to unknown reasons. Only $38.40 \%$ cases were diagnosed before delivery.

There are only few studies conducted in Pakistan on nuchal cord. ${ }^{19,24-26}$ Whereas, a large number of studies conducted in developed countries pertaining to nuchal cord. ${ }^{1-18}$ The presence of nuchal cord associated with adverse fetaloutcomes like preterm deliveries, low birth weight, fetal distress, rarely perinatal mortality and variable fetal heart rate decelerations as depicted in some studies..$^{2-7}$ But there was not found any association between perinatal complications and nuchal cord in other studies.

Nuchal cord is understood to reason fetal cardiac arrhythmia and acidosis, but at 1 minute \& 5 minute APGAR score is not much affected. A local study ${ }^{19}$ did not show difference significantly in mean 1-minute Apgar score among two groups, although the group of nuchal cord did tend to have a large infants percentage born with Apgar score of $<7$. At five minutes after birth this difference was absent, so it is proposed that nuchal cord effect on the fetus is temporary with no long-term effects. This finding also supported by other studies ${ }^{27,28}$ Likewise in our study adverse fetal outcomes, i.e. fetal heart rate irregularities were found in $18.29 \%$ cases, apgar score of $<7$ at 1 minute and $<7$ at 5 minutes were $28.22 \%$ and $09.64 \%$ respectively. 
A local study ${ }^{19}$ reported, it is important to note that APGAR score in group of nuchal cord of this study were comparable to those of the control group, despite of the fact that during labour high occurrence of fetal distress noted. It may be that the Apgar score is a better health sign of newborn at the time of birth than the variations in heart rate noted during labor. Most research examining effects associated with a nuchal twine at delivery become aware of an affiliation with fetal coronary heart charge abnormalities, operative shipping, decrease cord ph, lower Apgar scores and admission to special care nursery. $2,4,12,20$

In a local study ${ }^{19}$ eleven neonates with cord around neck needs admission in intensive care unit while in group B only 3 neonates needed admission to intensive care unit which shows that high neonatal morbidity in nuchal cord cases as compare to cases without nuchal cord. In our study admission to neonatal intensive care unit was found in $36.31 \%$ cases, which shows higher neonatal morbidity in our cases, may be due to very late presentation of cases to specialized centers or tertiary care hospitals.

In a retrospective population based study conducted by Sheiner and colleagues ${ }^{5}$ confirmed that there is no association of nuchal cord with adverse perinatal outcomes. While in a local study, ${ }^{19}$ in group A of deliveries over $26.4 \%$ were complicated due to some form of meconium staining whereas, meconium was present in $6.6 \%$ cases of group B, which become different among the results of Spellacy et $\mathrm{al}_{,}{ }^{27}$ that meconium incidence is not enhance by nuchal cords. Similarly in our study meconium stained liquor was recorded in $\mathbf{2 8 . 5 \%}$ cases which is in accordance with the results of above cited local study. ${ }^{19}$

In a local study, ${ }^{19}$ there has been determined no association among twine round neck and maternal age. There was no impact of nuchal cord on the period of pregnancy as reported in other studies. ${ }^{19,27}$ Results of our study also in accordance with the above mentioned studies that maternal age was not associated with nuchal cord presence as it is evidence from our study that all maternal age groups were having neonates with nuchal cords.

In a local study ${ }^{19}$ in group with cord around neck $(26.7 \%)$ the incidence of emergency lower segment caesarian section was more as compared to the group without nuchal cord $(23.4 \%)$. They found that with and without nuchal cord there was no difference in mode of deliveries. In those pregnancies without nuchal cord, the rate of instrumental deliveries was much common, while it is contentious in literature. ${ }^{27,28}$ In our study normal vaginal delivery was done in majority of cases $(50.49 \%)$, cesarean section in $36.87 \%$ cases, instrumental deliveries in $12.05 \%$ cases and breech deliveries in $0.56 \%$ cases. In another study, Rhoades and colleagues ${ }^{20}$ reported that the prevalence of a nuchal cord changed into lower at planned caesareans in contrast to emergency caesareans or vaginal births suggesting that perhaps a few babies turn out to be entangled of their cords in the course of labour.

The fewer studies were unfit to locate any critical results related with nuchal cords.,29 Sheiner and colleagues ${ }^{5}$ reported that lesser perinatal death rate in babies with nuchal cords and Mastrobattista and colleagues ${ }^{6}$ also discovered a lower cesarean rate in babies with a nuchal cord. Accordingly the lower cesarean rate may support Rhoades and colleagues ${ }^{20}$ discoveries and the hypothesis that a few infants wind up caught in their nuchal amid work.

\section{CONCLUSIONS}

It is concluded on the basis of our results that frequency of nuchal cord was found not higher than reported in the literature. Maximum cases were detected at the time of delivery. However, pre-birth location of string around neck through ultrasound can help the obstetrician to be more mindful to screen the work of these patients with string around neck. Our results showed minimum adverse fetal outcomes. Normal vaginal delivery was the main mode of delivery and these findings help obstetrician in counseling parents with this condition who may be scared unnecessarily. Copyright $\odot 15$ Oct, 2018. 


\section{REFERENCES}

1. Meena P, Meena PBS, Kashyap M, Meena K. Fetomaternal outcome in primigravida having single loop of cord round the neck. Intnl J Open Sci Res J Dentl Med Sci 2015; 14;7-12.

2. Schaffer L, Burkhardt T, Zimmermann R, Kurmanavicius $J$. Nuchal cords in term and postterm deliveriesedo we need to know? Obstet Gynecol 2005; 106:23-8.

3. Henry E, Andres RL, Christensen RD. Neonatal outcomes following a tight nuchal cord. J Perinatol 2013; 33:231-4.

4. Clapp $3^{\text {rd }}$ JF, Stepanchak W, Hashimoto K, Ehrenberg $\mathrm{H}$, Lopez $\mathrm{B}$. The natural history of antenatal nuchal cords. Am J Obstet Gynecol 2003; 189:488-93.

5. Sheiner E, Abramowicz JS, Levy A, Silberstein T, Mazor $M$, Hershkovitz R. Nuchal cord is not associated with adverse perinatal outcome. Arch Gynecol Obstet 2006; 274:81-3.

6. Mastrobattista JM, Hollier LM, Yeomans ER, Ramin SM, Day MC, Sosa A, et al. Effects of nuchal cord on birthweight and immediate neonatal outcomes. Am J Perinatol 2005; 22:83-5.

7. Peregrine E, O'Brien P, Jauniaux E. Ultrasound detection of nuchal cord prior to labor induction and the risk of Cesarean section. Ultrasound Obstet Gynecol 2005; 25:160-4.

8. Narang Y, Vaid NB, Jain S, Suneja A, Guleria K, Faridi $\mathrm{MM}$, et al. Is nuchal cord justified as a cause of obstetrician anxiety? Arch Gynecol Obstet 2014; 289:795-801.

9. Liu LC, Wang YC, Yu MH, Su HY. Major risk factors for stillbirth in different trimesters of pregnancyea systematic review. Taiwan J Obstet Gynecol 2014; 53:141-5.

10. Liu LC, Huang HB, Yu MH, Su HY. Analysis of intrauterine fetal demiseea hospital-based study in Taiwan over a decade. Taiwan J Obstet Gynecol 2013; 52:546-50.

11. Nkwabong E, Fomulu JN. Neonatal outcome in cases of nuchal cord in Cameroon. Int $\mathrm{J}$ Gynecol Obstet $2011 ; 114: 287-8$.

12. Nelson KB, Grether JK. Potentially asphyxiating conditions and spastic cerebral palsy in infants of normal birth weight. Am J Obstet Gynecol 1998; 179:507-13.

13. Hasegawa J, Matsuoka R, Ichizuka K, Sekizawa A, Okai T. Ultrasound diagnosis and management of umbilical cord abnormalities. Taiwan J Obstet Gynecol 2009; 48:23-7.

14. Kong CW, Lee DH, Chan LW, To WW. Impact of nuchal cord on fetal outcomes, mode of delivery, and management: a questionnaire survey of pregnant women. Hong Kong Med J 2015; 21:143-8.

15. Mercer JS, Skovgaard RL, Peareara-Eaves J, Bowman TA. Nuchal cord management and nurse-midwifery practice. J Midwifery Womens Health 2005; 50:373-9.

16. Iffy L, Varadi V, Papp E. Untoward neonatal sequelae deriving from cutting of the umbilical cord before delivery. Med Law 2001; 20:627:34.

17. Collins J. Tight nuchal cord morbidity and mortality. Am J Obstet Gynecol 1999; 180:251.

18. Schaefer M, Laurichesse-Delmas H, Ville $\mathrm{Y}$. The effect of nuchal cord on nuchal translucency measurement at 10-14 weeks. Ultrasound Obstet Gynecol 1998; 11:271-3.

19. Taizon S. Perinatal outcome in cases with or without nuchal cord. Ann King Edward Med Uni 2014; 20:15964.

20. Rhoades DA, Latza U, Mueller BA. Risk factors and outcomes associated with nuchal cord: A populationbased study. J Reprod Med 1999; 44:39-45.

21. Kumaria S, Saxena A, Monga D, Malik A, Kabra M, Kurray RM. Significance of cord problems at birth. Indian Pediatr 1992; 29:301-5.

22. Dhar KK, Ray SN, Dhall GI. Significance of nuchal cord. J Indian Med Assoc 1995; 93:451-3.

23. Greenwood C, Impey L. The association of nuchal cord with cerebral palsy is influenced by recording bias. Early Hum Dev 2002; 68:15-9.

24. Sharif S, Sultana S, Waqar F, Saeed A, Sadia S. Maternal and fetal outcome of pregnancies with umbilical cord problems. J Islamic Int Med Coll 2011; 6:14-8.

25. Sajjad R, Mushtaq M, Mustafa N. Cord around neck in singleton term pregnancies and its outcome. Pak Armed Forces Med J 2014; 64:51-5.

26. Zahoor F, Minhas Z, Zaki A. Perinatal outcome of nuchal cord. J Postgrad Med Inst 2013; 27:174-8.

27. Spellacy WN, Greuem H, Fisch RO. The umbilical cord complications of true knots, nucha coils, and cords around the body. Am J Obstet Gynaecol 1966; 94:1136-42.

28. Weber T. The influence of cord complications on fetal 
ph, neonatal Apgar scores, and the acid base state and oxygenation of the umbilical artery and vein. $J$ Perinet Med 1981; 9:134-9.
29. Gonzalez - Quintero VH, Tolamat L, Muller AC, O'Sullivan MJ, Martin D. Outcome of pregnancies with sonographically detected nuchal cords remote from delivery. J Ultasound Med 2004; 23:43-7.

\title{
POVERTY IS THE WORST FORM OF VIOLENCE.
}

\author{
"Mahatma Gandhi"
}

\section{AUTHORSHIP AND CONTRIBUTION DECLARATION}

\begin{tabular}{|c|l|l|l|}
\hline Sr. \# & Author-s Full Name & \multicolumn{1}{|c|}{ Contribution to the paper } & Author=s Signature \\
\hline 1 & Abida Nasreen & $\begin{array}{l}\text { Writing of manuscript, data } \\
\text { collection and compiling resutls. } \\
\text { Review the article. }\end{array}$ \\
\hline 2 & Hina Niaz & R & \\
\hline
\end{tabular}

